\title{
Use of clonidine following the weaning phase of the elderly patients underwent elective on-pump cardiac surgery: a prospective randomized study
}

\author{
S Caroleo ${ }^{1 *}$, F Onorati $^{2}$, O Bruno ${ }^{1}$, D Vuoto $^{1}$, F Infelise ${ }^{1}$, A Rubino $^{2}$, E Santangelo $^{3}$, A Renzulli ${ }^{2}$, B Amantea ${ }^{1}$ \\ From de Senectute: Age and Health Forum \\ Catanzaro, Italy. 5-7 December 2009
}

\section{Background}

Alpha-2 adrenergic agonists reduce mortality and myocardial infarction following vascular and cardiac surgery [1]. Few data exist about the weaning phase in this setting.

\section{Materials and methods}

Design: Analysis of a prospective and randomized collected database.

Setting: Intensive Care Unit (ICU) in an University Hospital.

Patients: A total of 45 patients aged $>65(26 \mathrm{M}, 19 \mathrm{~F}$; ASA II-III) submitted to elective on-pump cardiac surgery from November 2007 to June 2008.

Randomization: On ICU admission the patient was assigned to group 1 (Clonidine, intervention group) or group 2 (Placebo, control group).

Interventions: Group 1 received intravenous (IV) bolus of Clonidine $0.5 \mathrm{microg} / \mathrm{kg}$ followed by continuous infusion of $1-2 \mathrm{microg} / \mathrm{kg} / \mathrm{hr}$ all over the weaning protocol phase. Group 2 received IV continuous infusion of Sodium Chloride $0.9 \%$ all over the weaning phase.

Data collection: We evaluated hemodynamic parameters, Troponin I (TnI) blood levels, weaning parameters, Delirium Detection Score (DDS), weaning duration and ICU length of stay. The patients were evaluated preoperatively (T0), on ICU admission (T1), after 6 hours (T2) and 30 minutes after the start of weaning protocol (T3).

\section{Results}

No differences in preoperative and operative variables ( $\mathrm{p}=\mathrm{NS}$ for all measurements). The incidence of postoperative atrial fibrillation was lower in group $1(\mathrm{p}<0.001)$.
Following the weaning phase, Heart Rate, Mean Pulmonary Arterial Pressure and Pulmonary Arterial Occlusion Pressure were lower in group 1 (respectively $\mathrm{p}<0.001$, $\mathrm{p}=0.019$ and $\mathrm{p}=0.037$ ). The $\mathrm{TnI}$ levels was lower in group $1(\mathrm{p}=0.05)$. The ratio of respiratory rate and tidal volume $(\mathrm{RR} / \mathrm{TV})$ and the product of $R R$ and pressure support (RR $\times$ PS) were lower in group 1 (both $\mathrm{p}<0.001$ ); the ratio of $\mathrm{PaO}_{2}$ and $\mathrm{FIO}_{2}$ (PA/FI) and $\mathrm{PaCO}_{2}$ were higher in group 1 (respectively $\mathrm{p}=0.0035$ and $\mathrm{p}<0.001$ ). DDS was lower in group 1 ( $\mathrm{p}=0.0028)$. Weaning duration and ICU length of stay were similar in the two groups $(\mathrm{p}=\mathrm{NS})$.

\section{Conclusions}

The use of Clonidine in this setting reduce the stressresponse during the weaning phase, improving hemodynamic stability and myocardial protection.

\section{Author details}

'Deparment of Anaesthesia and Reanimation, University of Catanzaro, Catanzaro, Italy. ${ }^{2}$ Department of Cardiac Surgery, University of Catanzaro, Catanzaro, Italy. ${ }^{3}$ Department of Anaesthesia and Oncological Intensive Care, Italy.

Published: 19 May 2010

\section{Reference}

1. Wijeysundera DN, Naik JS, Beattie WS: Alpha-2 adrenergic agonists to prevent perioperative cardiovascular complications: a meta-analysis. Am J Med 2003, 114(9):742-52.

doi:10.1186/1471-2318-10-S1-A97

Cite this article as: Caroleo et al.: Use of clonidine following the weaning phase of the elderly patients underwent elective on-pump cardiac surgery: a prospective randomized study. BMC Geriatrics 2010 10(Suppl 1):A97. 